\title{
The role and mechanism of IncRNA NEAT1 in the fibrosis of pulmonary epithelial cell
}

\author{
Hui Xu ${ }^{1} \cdot$ YanBo Chen ${ }^{2} \cdot$ Jinqiang Zhuang ${ }^{1} \cdot$ Shun $\mathrm{Zhu}^{1} \cdot$ Bing $\mathrm{Xu}^{3} \cdot$ Jiang Hong ${ }^{1}$ (i)
}

Accepted: 18 December 2019 / Published online: 19 February 2020

(c) The Author(s) 2020

\begin{abstract}
Background Pulmonary fibrosis is a serious clinical fatal disease. Epithelial-mesenchymal transition (EMT) and lncRNA NEAT1 have been implied in its development and progression.

Objective To study the role of lncRNA NEAT1 in the progression of fibrosis in human pulmonary epithelial cells (BEAS2B). Specifically, BEAS-2B was transfected with NEAT1 and miR-29c, EMT and cell proliferation were measured and the expression level of relevant genes was determined by Western blot.

Result Results showed that NEAT1 promotes fibrosis and proliferation of BEAS-2B cells via the up-regulation of $\alpha$-SMA, Vimentin, Snail and proliferation-related genes including Cyclin D1 and Cyclin E; miR-29c is a target gene of NEAT1 and through which NEAT1 regulates EMT and expression of proliferation-related genes.

Conclusion This study investigated the mechanism of pulmonary fibrosis progression by elucidating the role of NEAT1/ miR-29c in the fibrosis and proliferation of BEAS-2B cells, thus providing a basis for the new therapeutic targets of pulmonary fibrosis.
\end{abstract}

Keywords LncRNA NEAT1 $\cdot$ Fibrosis $\cdot$ Epithelial-mesenchymal transition $\cdot$ Proliferation $\cdot$ miR-29c

\section{Introduction}

Pulmonary fibrosis is a serious clinical fatal disease with limited effective drugs. The mechanism of its pathogenesis and progression is complicated. Nonetheless, it is generally believed that epithelial-mesenchymal transition (EMT) plays an important role in the development and progression of pulmonary fibrosis (Horowitz and Thannickal 2006;

Hui $\mathrm{Xu}$ and YanBo Chen have contributed equally to this work.

Bing Xu

saint_xue@msn.com

$\triangle$ Jiang Hong

jhong.pku@163.com

1 Department of Internal and Emergency Medicine, Shanghai General Hospital, Shanghai Jiao Tong University School of Medicine, Shanghai, China

2 Department of Urology, Shanghai Ninth People's Hospital Affiliated to Shanghai Jiaotong University School of Medicine, Shanghai, China

3 Department of Emergency, Shanghai Ninth People's Hospital Affiliated to Shanghai Jiaotong University School of Medicine, Shanghai, China
Rout-Pitt et al. 2018). Furthermore, the activation of several signaling pathways such as transforming growth factorbeta (TGF-beta) and Wnt/beta-catenin signaling pathways were suggested to play an important role in the EMT process (Willis and Borok 2007; Saito et al. 2018; Juan et al. 2017). Therefore, the investigation of EMT and its participating factors may provide new approaches for targeted treatment of pulmonary fibrosis.

Long non-coding RNAs (lncRNA) are a class of long RNA transcripts without protein-coding ability (Moran et al. 2012; Lee et al. 2019). In recent years, lncRNA has been found to be involved in the biological processes of many diseases by regulating a variety of cell processes such as cell cycle, apoptosis, metabolism, and EMT (Zhang et al. 2019; Guo et al. 2019). Naturally, aberrantly expressed IncRNA was thought to be one of the important biomarkers for various diseases including pulmonary fibrosis. For example, Sun found that overexpressed lncRNA uc.77 and 2700086A05Rik were involved in the pathogenesis of idiopathic pulmonary fibrosis via its regulation on EMT (Sun et al. 2016). And Song explored the role of lncRNA MRAK088388 and MRAK081523 and the corresponding mechanisms involved in pulmonary fibrosis (Song 
et al. 2014). Studies have also shown that lncRNA NEAT1 accelerated the progression of liver fibrosis by modulating miR-122 and Kruppel-like factor 6 (Yu et al. 2017), which, however, has not been validated in the progression of pulmonary fibrosis. Therefore, in this study, we investigated the role of lncRNA NEAT1 in the development and progression of pulmonary fibrosis in vitro, as well as its underlying mechanisms.

\section{Materials and methods}

\section{Cell culture and treatment}

Human normal pulmonary epithelial cells (BEAS-2B) were purchased from FuHeng Cell Center, Shanghai, China, and cultured using LHC-8 (Gibco) medium at $37{ }^{\circ} \mathrm{C}$ with $5 \%$ $\mathrm{CO}_{2}$. According to the method used by Liu et al. (2017a), the pulmonary fibrosis cell model was established by inducing the cells with TGF- $\beta 1$ at a concentration of $5 \mathrm{ng} / \mathrm{ml}$ for $48 \mathrm{~h}$.

\section{Transfection}

Transfections of the NEAT1 expression plasmid, NEAT1 siRNA, miR-29c mimics and miR-29c inhibitor were performed using Lipofectamine 2000 reagent (Thermo Fisher Scientific). Specifically, the full-length NEAT1 sequence was inserted into the pcDNA3.1 vector for construction of NEAT1 expression plasmid and the target sequence of the NEAT1 siRNA was: 5'-GUGAGAAGUUGCUUAGAAACU UUCC-3' (Wang et al. 2016).

\section{qRT-PCR}

Total RNA was extracted using the RNAiso Plus kit (Takara). Reverse transcription of RNA was performed using TM RT Master Mix (Takara), and qPCR was performed using SYBR Premix Ex Taq II (Takara). The qRT-PCR reaction was performed on an ABI 7900 platform (Applied Biosystems). mRNA expression was determined with $\beta$-actin as an internal reference and the relative expression level of the target gene was calculated using the $2^{-\Delta \Delta \mathrm{CT}}$ method. Primer sequences are as follows: NEAT1-F: TTGTTCCAG AGCCCATGAT; NEAT1-R: TGAAAACCTTTACCCCAG GA; $\beta$-actin-F: GGCTCCGGCATGTGCAAG; $\beta$-actin-R: CCTCGGTCAGCAGCACGG.

\section{Cell proliferation measured with CCK-8 assay}

BEAS-2B cells were seeded in 96-well plates for treatment and transfection. The cell proliferation was determined with the CCK8 kit (Dojindo, Japan) at three different time points ( $24 \mathrm{~h}, 48 \mathrm{~h}$, and $72 \mathrm{~h}$ ) after the transfection where the absorbance at $450 \mathrm{~nm}$ was measured by a microplate reader.

\section{Western blot}

The total protein was extracted from cells with RIPA lysis buffer and the protein concentration was determined using a BCA kit. $20 \mu \mathrm{g}$ of protein was loaded into the SDS-PAGE gel for electrophoresis, and the protein was then electroporated into the PVDF membrane for blocking and subsequent primary and secondary antibody incubation. Exposure and capture of images were conducted using a chemiluminescent imaging system (Clinx Science Instruments). Primary antibodies were purchased from Abcam and beta-actin was used as an internal reference. The antibodies used in this experiment were: $\alpha$-SMA $(1: 1000$, ab32575, Abcam); Vimentin (1:1000, ab92547, Abcam); Snail (1:1000, ab53519, Abcam); Cyclin D1 (1:2000, ab16663, Abcam); Cyclin E (1:500, ab133266, Abcam); $\beta$-actin (1:2000, ab8226, Abcam).

\section{Construction of dual-luciferase reporter system}

The oligonucleotide (wt) containing the NEAT1 target sequence and its mutant sequence (mut) without the miR29c-binding site was amplified and cloned into the pmirGLO vector (luciferase reporter plasmid). After that, the luciferase reporter plasmid and miR-29c mimics were co-transfected into BEAS-2B cells. Luciferase activity was then measured using the dual-luciferase reporter assay system (Promega) according to the instructions.

\section{Statistical analysis}

Data were analyzed using SPSS 18.0 software and expressed as mean \pm SD. $p<0.05$ was considered statistically significant.

\section{Results}

\section{NEAT1 promoted fibrosis and proliferation of BEAS-2B cells}

First, we transfected BEAS-2B cells with NEAT1 overexpression plasmid and siRNA separately and verified by qRT-PCR to confirm the validity of overexpression plasmid (Fig. 1a) and siRNA (Fig. 1b). Following that, we then transfected NEAT 1 siRNA in TGF- $\beta 1$-induced BEAS2B cells, transfected BEAS-2B cells with NEAT1 overexpression plasmid, and cell morphology was observed under a microscope. The results showed that NEAT1 siRNA was able to reverse TGF- $\beta 1$-induced BEAS-2B 


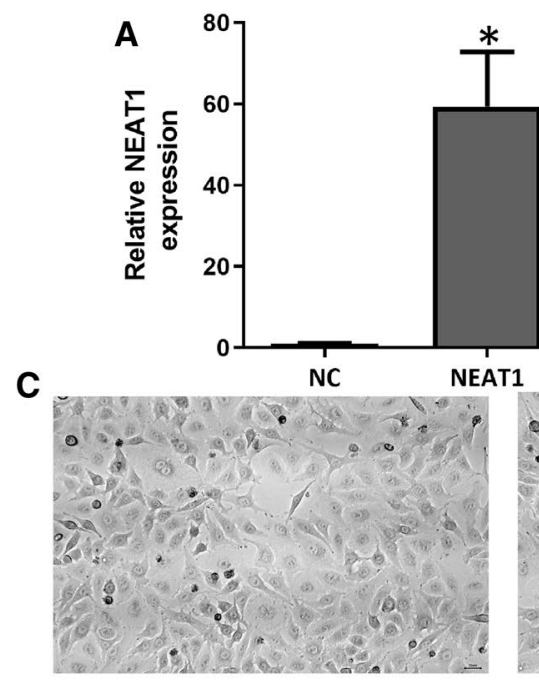

Normal

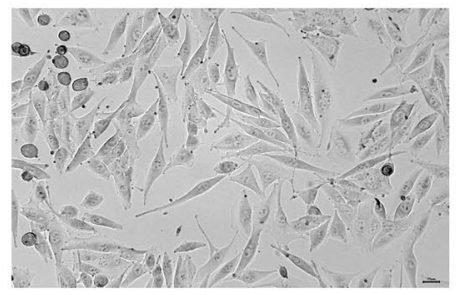

TGF- $\beta 1$

D

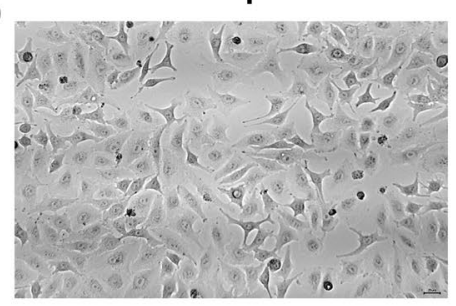

E
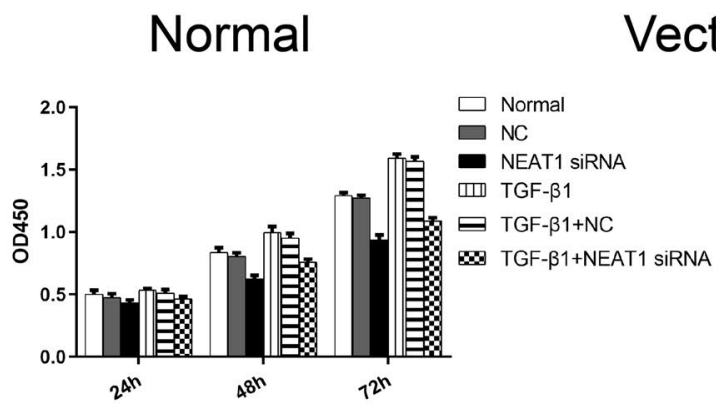
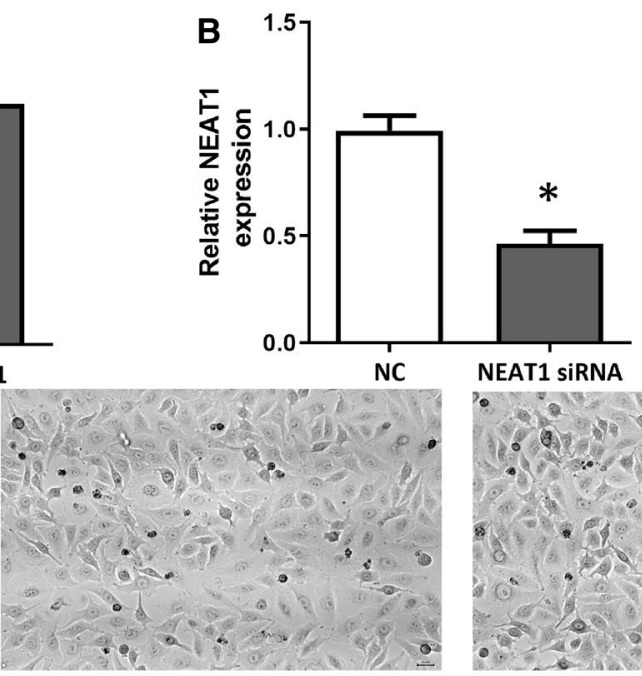

NC

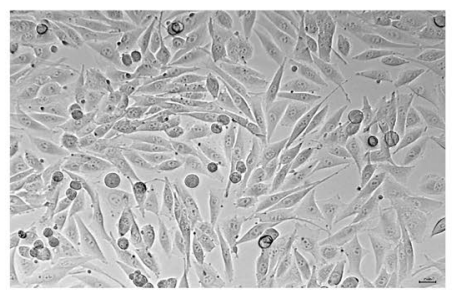

TGF- $\beta 1+N C$

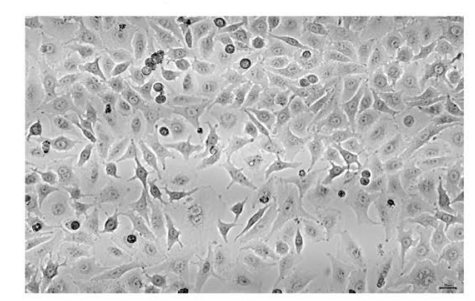

Vector

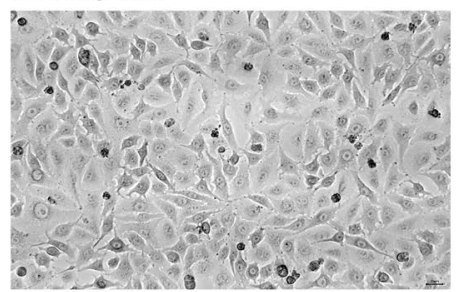

NEAT1 siRNA

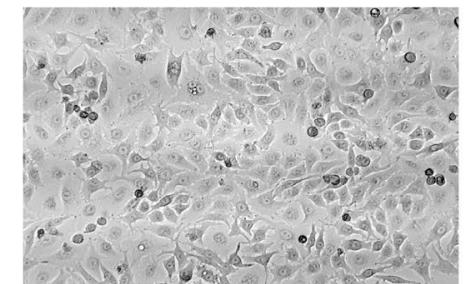

TGF- $\beta 1+N E A T 1$ siRNA

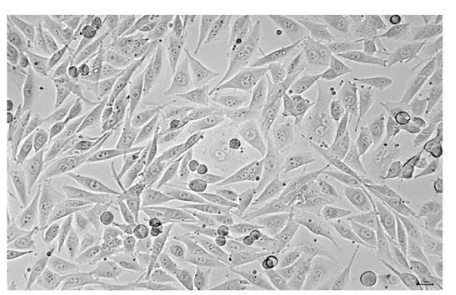

NEAT1

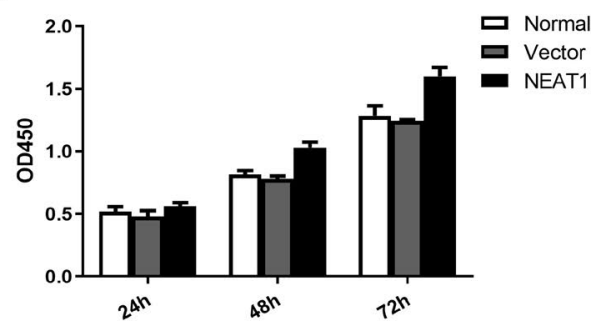

Fig. 1 NEAT1 promoted the fibrosis and proliferation of BEAS-2B cells. a, b qRT-PCR was used to verify NEAT1 overexpression and silencing in BEAS-2B cells; $\mathbf{c}$, $\mathbf{d}$ cell morphology was observed in

cell fibrosis (Fig. 1c), whereas overexpression of NEAT1 promoted BEAS-2B cell fibrosis (Fig. 1d). At the same time, the CCK8 assay demonstrated that NEAT1 siRNA inhibited the proliferation of TGF- $\beta 1$-treated BEAS-2B cells $(p<0.05)$ (Fig. 1e), while overexpression of NEAT1 promoted the proliferation of BEAS-2B cells $(p<0.05)$ different treatment groups; e, f CCK8 assay was used to detect cell proliferation activity in different treatment groups $(* p<0.05)$

(Fig. 1f). Taken together, these results indicated that NEAT1 promoted the fibrosis and proliferation of BEAS$2 \mathrm{~B}$ cells. 


\section{NEAT1 regulated the expression of EMT- and proliferation-related genes in BEAS-2B cells}

With the Western blot analysis, we found that TGF- $\beta 1$ induced the up-regulation of $\alpha$-SMA, Vimentin, Snail and proliferation-related genes such as Cyclin D1 and Cyclin E, while NEAT1 siRNA could reverse the up-regulation of these genes (Fig. 2). Not surprisingly, overexpression of NEAT1 promoted the up-regulation of these genes (Fig. 2). These results indicated that NEAT1 promotes the ibrosis and proliferation of BEAS-2B cells by promoting the expression of EMT- and proliferation-related genes.

\section{miR-29c is the target gene of NEAT1}

Using the StarBase database, we found that miR-29c is a potential target for NEAT1 (Fig. 3). Luciferase experimental results showed that overexpression of miR-29c inhibited the luciferase activity of the wt reporter plasmid and had no
Target site: chr11:65195314-65195335[+]

NEAT1 5’ ugcCUGUAUGUAAAUGGUGCUg 3’

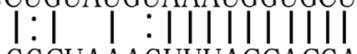
miR-29c 3' auuGGCUAAAGUUUACCACGAu 5'

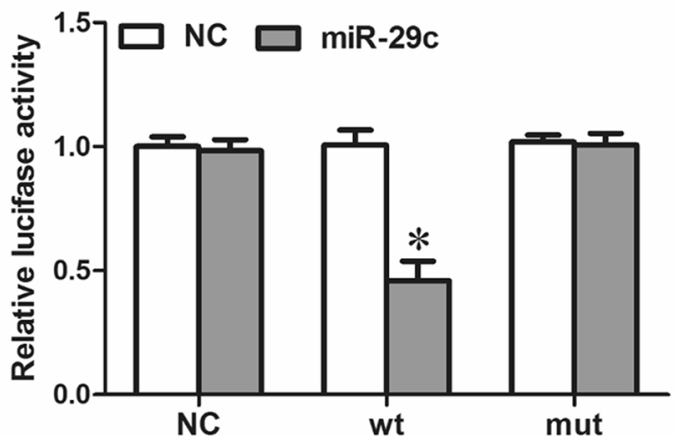

Fig. 3 Verification of luciferase reporter gene and the target site between NEAT1 and miR-29c $(* p<0.05)$
Fig. 2 NEAT1 regulated EMTand proliferation-related genes in BEAS-2B cells. a, c Western blot was used to detect the changes of genes involving in epithelial-mesenchymal transition and proliferation in each group; b, d Western blot bands were quantified; $* p<0.05$
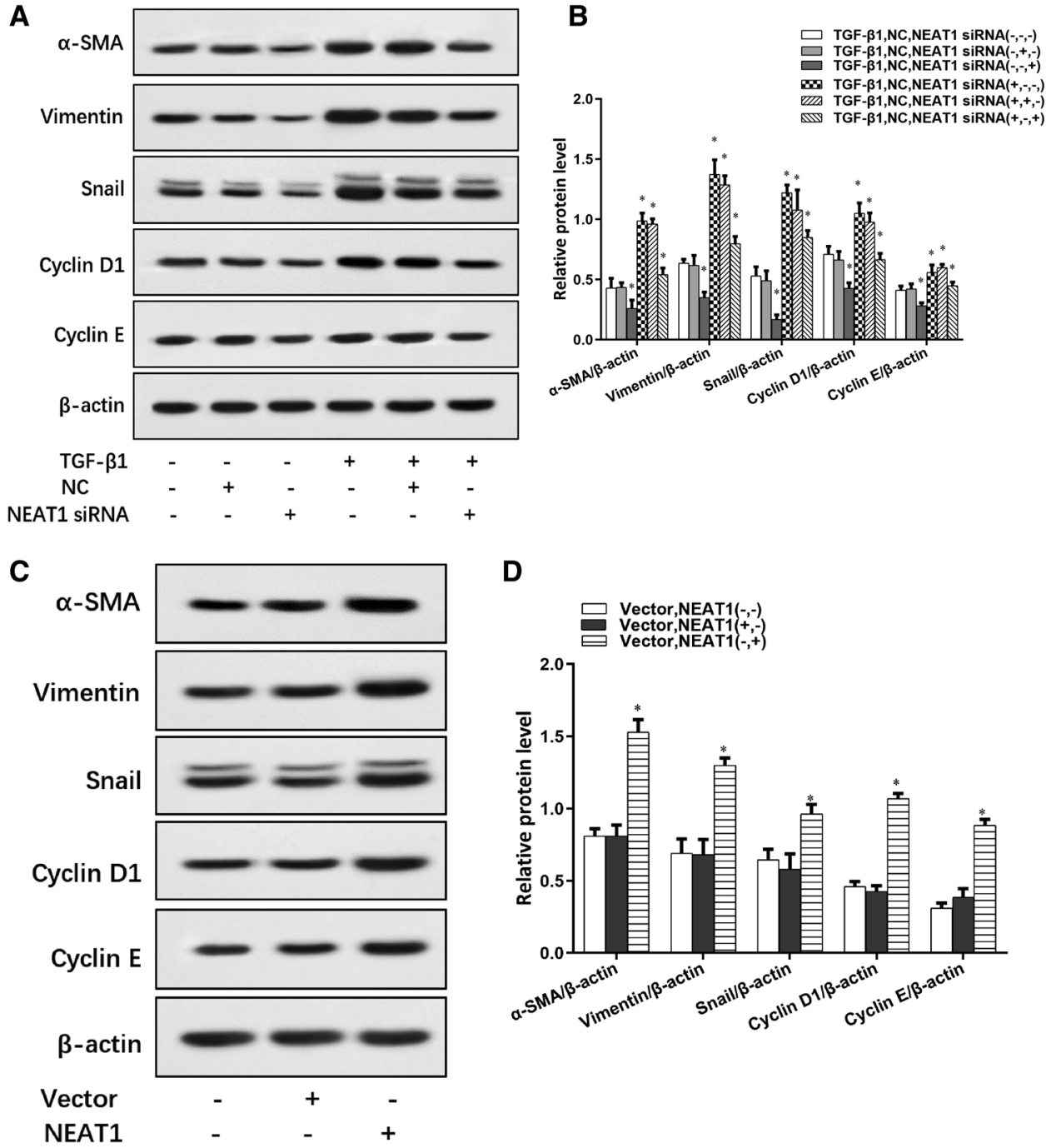
effect on the luciferase activity of the mut reporter plasmid (Fig. 3), indicating that miR-29c is a target gene of NEAT1.

\section{NEAT1 regulated BEAS-2B cell fibrosis and proliferation via miR-29c}

Recovery experiments showed that miR-29c inhibitor reversed the inhibition of fibrosis and proliferation induced by NEAT1 siRNA in BEAS-2B cells (Fig. 4a, b) $(p<0.05)$; on the contrary, miR-29c mimics reversed the fibrosis and proliferation-promoting effects induced by overexpression of NEAT1 (Fig. 4c, d) $(p<0.05)$. These results indicated that NEAT1 regulated BEAS-2B cell fibrosis and proliferation via miR-29c.

\section{NEAT1 regulated EMT- and proliferation-related genes through miR-29c}

Similarly, the recovery experiments also showed that miR29c inhibitor reversed the down-regulation of EMT- and proliferation-related genes induced by NEAT1 siRNA in BEAS-2B cells (Fig. 5a), whereas miR-29c mimics reversed the up-regulation of EMT- and proliferation-related genes caused by overexpression of NEAT1 (Fig. 5b). These lines of evidence indicated that NEAT1 regulated EMT- and proliferation-related genes through miR-29c.

\section{Discussion}

LncRNA and miRNA are hot topics in recent years. Compared with miRNA, there are relatively few studies on lncRNA and, therefore, many functions are still poorly understood. Nonetheless, studies have shown that lncRNA is actually involved in the progression of many diseases (Neil et al. 2019; Prinz et al. 2019). LncRNA NEAT1 has been implicated in various tumors. For example, HIF- $2 \alpha$-activated lncRNA NEAT1 promoted the invasion and metastasis of hepatocellular carcinoma cells by affecting EMT (Zheng et al. 2018); the RGFR pathway regulated lncRNA NEAT1 and promoted the progression of glioblastoma by regulating EZH2 and Wnt/ $\beta$-cantenin pathways (Chen et al. 2017). Moreover, NEAT1 was reported to be involved in liver fibrosis (Yu et al. 2017) and renal fibrosis (Huang et al. 2019); however, there is only few research on NEAT1 in pulmonary fibrosis. Therefore, in this regard, our research is both innovative and theoretically founded.

Fig. 4 NEAT1 regulated BEAS2B cell fibrosis and proliferation via miR-29c. a, b cell morphologies in different treatment groups; $\mathbf{c}, \mathbf{d}$ cell proliferation activities in different treatment groups detected by CCK8 assay

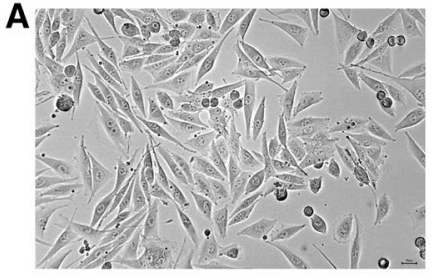

TGF- $\beta+$

C

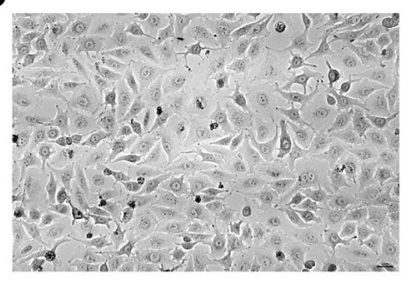

NC

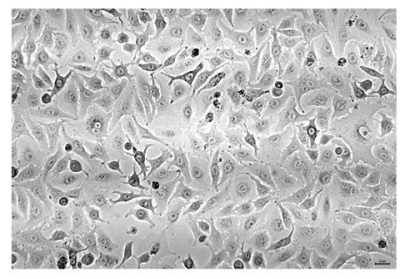

TGF- $\beta+$ NEAT1 siRNA

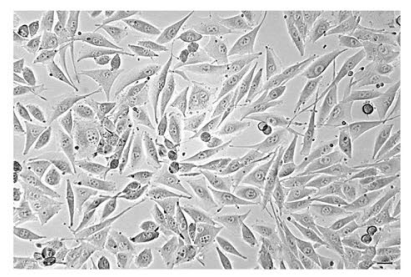

NEAT1

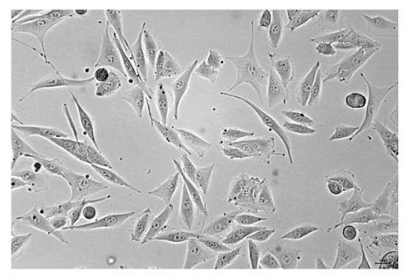

TGF- $\beta+N E A T 1$ siRNA + miR-29c inhibitor

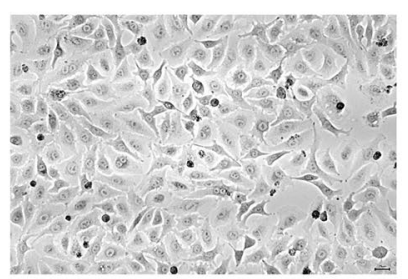

NEAT1+miR-29c mimics
B

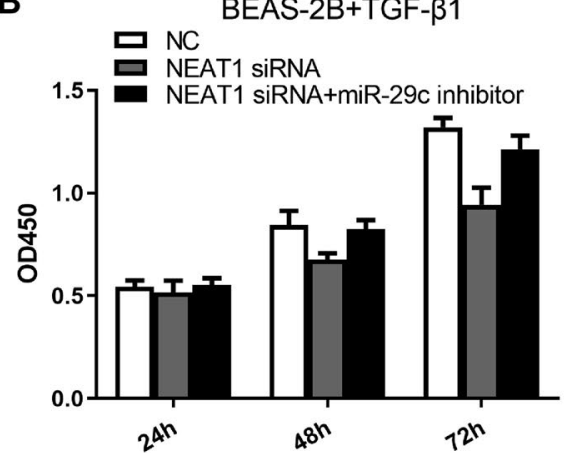

D

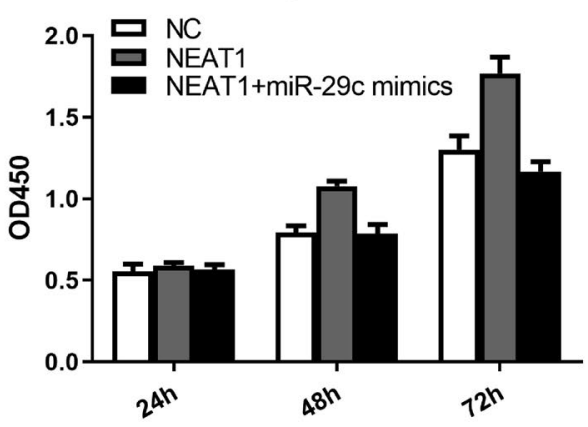


Fig. 5 NEAT1 regulated EMTand proliferation-related genes via miR-29c. a, c Western blot was used to detect the expression of epithelial-mesenchymal transition and proliferationrelated genes in each group; b, $\mathbf{d}$ Western blot bands were quantified; $* p<0.05$

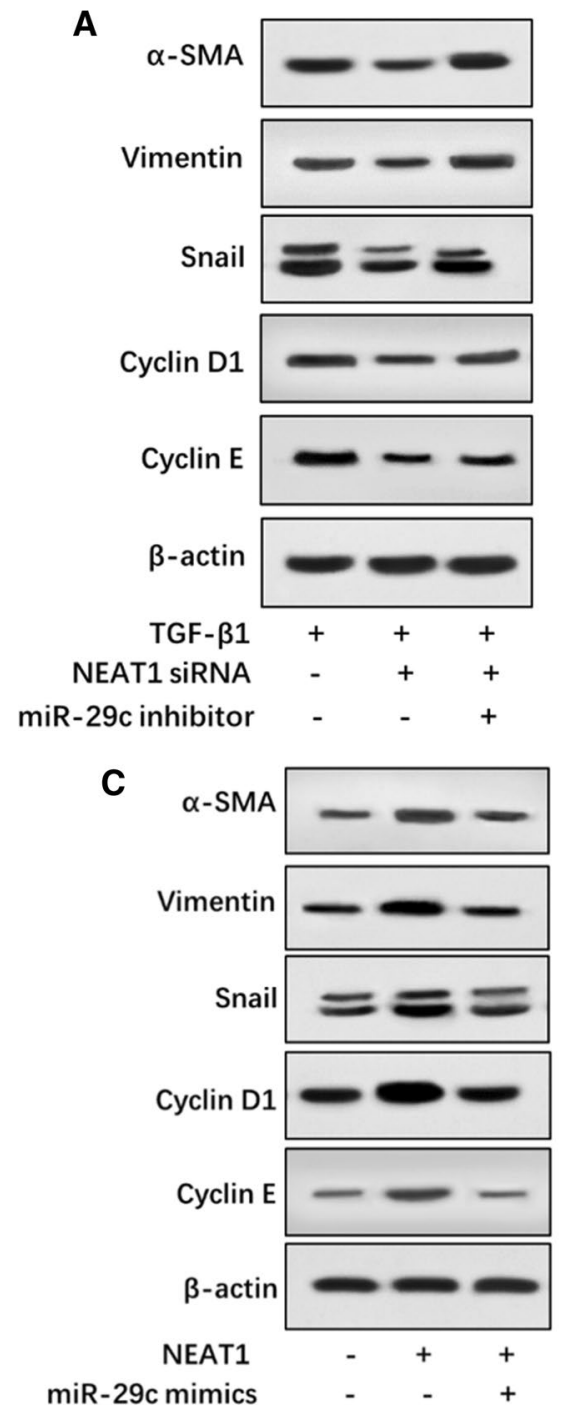

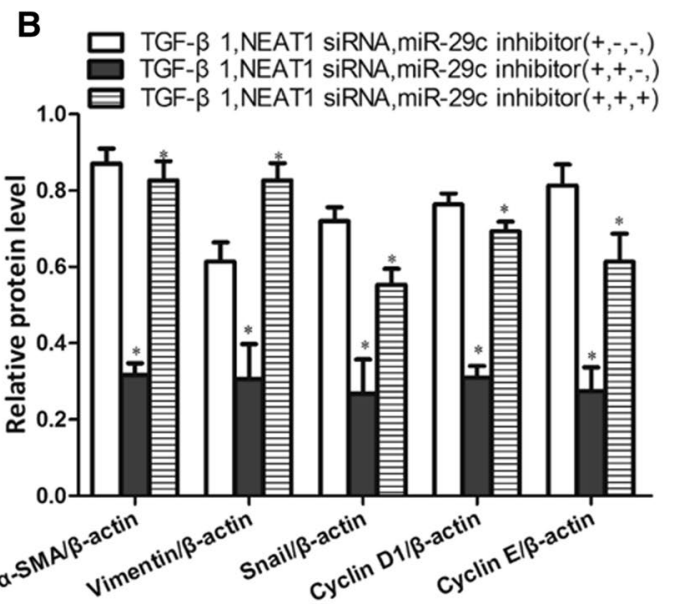

Pulmonary fibrosis is a serious disease characterized by fibroblast proliferation, and EMT is one of the main sources of fibroblasts. During this process, epithelial cells lose their epithelial phenotype, acquire fibroblast-like properties, and exhibit decreased cell adhesion and increased motility. At the molecular level, this process is accompanied by an increase of markers for pulmonary fibrosis, such as $\alpha$-SMA, Vimentin, and Snail (Liu et al. 2017b). The results of this study indicated that NEAT1 promoted fibrosis and proliferation of BEAS-2B cells, which was due to the up-regulation of EMT-related genes including $\alpha$-SMA, Vimentin, Snail and proliferation-related genes such as Cyclin D1 and Cyclin E. In other words, we believe that NEAT1 can promote the progression of pulmonary fibrosis.

LncRNA can be involved in related physiological and pathological processes by acting as a ceRNA for microRNA (Cui and Zhao 2019; Huang et al. 2019). In this study, we found that miR-29c is the target gene of NEAT1. Xie et al. (2017) found that miR-29c could prevent pulmonary fibrosis by regulating epithelial cell renewal and apoptosis; a study by Matsushima and Ishiyama (2016) showed that microRNA-29c regulated apoptosis by modulating the cell surface death receptor Fas of lung fibroblasts, which in turn inhibiting pulmonary fibrosis. Therefore, in these studies, miR-29c acted as an inhibitor in the process of pulmonary fibrosis. Our results demonstrated that NEAT1 regulated the expression of EMT- and proliferation-related genes through miR-29c to affect the fibrosis and proliferation of BEAS-2B cells, which substantiated the miR-29c regulation network in pulmonary fibrosis.

In summary, our study showed that NEAT1 affected the fibrosis and proliferation of BEAS-2B cells by regulating the expression of EMT- and proliferation-related genes through its target gene miR-29c, which in turn, rendering NEAT1 as a potential target for the treatment of pulmonary fibrosis. 
Author contribution Bing Xu and Jiang Hong designed experiments; Hui Xu and YanBo Chen carried out experiments and wrote the manuscript; Jinqiang Zhuang and Shun Zhu analyzed experimental results.

Funding This study was funded by Shanghai Jiaotong University Medical and Industrial Cross Project (YG2017QN06).

\section{Compliance with ethical standards}

We confirmed that all methods in our study were performed in accordance with the relevant guidelines of CONSORT 2010.

Conflict of interest The authors declare that they have no conflict of interest.

Open Access This article is licensed under a Creative Commons Attribution 4.0 International License, which permits use, sharing, adaptation, distribution and reproduction in any medium or format, as long as you give appropriate credit to the original author(s) and the source, provide a link to the Creative Commons licence, and indicate if changes were made. The images or other third party material in this article are included in the article's Creative Commons licence, unless indicated otherwise in a credit line to the material. If material is not included in the article's Creative Commons licence and your intended use is not permitted by statutory regulation or exceeds the permitted use, you will need to obtain permission directly from the copyright holder. To view a copy of this licence, visit http://creativecommons.org/licenses/by/4.0/.

\section{References}

Chen Q, Cai J, Wang Q et al (2017) Long non-coding RNA NEAT1, regulated by the EGFR pathway, contributes to glioblastoma progression through the WNT/ $\beta$-Catenin pathway by scaffolding EZH2. Clin Cancer Res 24(3):684-695

Cui H, Zhao J (2019) LncRNA TMPO-AS1 serves as a ceRNA to promote osteosarcoma tumorigenesis by regulating miR-199a-5p/ WNT7B axis. J Cell Biochem 121(3):2284-2293

Guo J, Liu Z, Gong R (2019) Long noncoding RNA: an emerging player in diabetes and diabetic kidney disease. Clin Sci 133:1321-1339

Horowitz J, Thannickal V (2006) Epithelial-mesenchymal interactions in pulmonary fibrosis. Semin Respir Crit Care Med 27(6):600-612

Huang S, Xu Y, Ge X et al (2019a) Long noncoding RNA NEAT1 accelerates the proliferation and fibrosis in diabetic nephropathy through activating Akt/mTOR signaling pathway. J Cell Physiol 234(7):11200-11207

Huang MJ, Zhao JY, Xu JJ et al (2019b) lncRNA ADAMTS9-AS2 controls human mesenchymal stem cell chondrogenic differentiation and functions as a ceRNA. Mol Ther Nucleic Acids 18:533-545

Juan S, Feng L, Meihui L et al (2017) Distinct roles of Wnt/ $\beta$-catenin signaling in the pathogenesis of chronic obstructive pulmonary disease and idiopathic pulmonary fibrosis. Mediat Inflamm 2017:1-16

Lee H, Zhang Z, Krause HM (2019) Long noncoding RNAs and repetitive elements: junk or intimate evolutionary partners? Trends Genet 35(12):892-902
Liu Y, Li Y, Xu Q et al (2017a) Long non-coding RNA-ATB promotes EMT during silica-induced pulmonary fibrosis by competitively binding miR-200c. Biochim Biophys Acta Mol Basis Dis 864(2):420-431

Liu H, Wang B, Zhang J et al (2017b) A novel lnc-PCF promotes the proliferation of TGF- $\beta 1$-activated epithelial cells by targeting miR-344a-5p to regulate map3k11 in pulmonary fibrosis. Cell Death Dis 8(10):e3137

Matsushima S, Ishiyama J (2016) MicroRNA-29c regulates apoptosis sensitivity via modulation of the cell-surface death receptor, Fas, in lung fibroblasts. Am J Physiol-Lung Cell Mol Physiol 311(6):L1050-L1061

Moran VA, Perera RJ, Khalil AM (2012) Emerging functional and mechanistic paradigms of mammalian long non-coding RNAs. Nucleic Acids Res 40:6391-6400

Neil CR, Fairbrother WG (2019) Intronic RNA: Ad'junk' mediator of post-transcriptional gene regulation. Biochim Biophys Acta Gene Regul Mech 1862:194439

Prinz F, Kapeller A, Pichler M et al (2019) The implications of the long non-coding RNA in non-cancerous diseases. Int J Mol Sci 20:627

Rout-Pitt N, Farrow N, Parsons D et al (2018) Epithelial mesenchymal transition (EMT): a universal process in lung diseases with implications for cystic fibrosis pathophysiology. Respir Res 19:136

Saito A, Horie M, Nagase T (2018) TGF- $\beta$ signaling in lung health and disease. Int J Mol Sci 19:2460

Song X, Cao G, Jing L et al (2014) Analysing the relationship between lncRNA and protein-coding gene and the role of lncRNA as ceRNA in pulmonary fibrosis. J Cell Mol Med 18(6):991-1003

Sun H, Chen J, Qian W et al (2016) Integrated long non-coding RNA analyses identify novel regulators of epithelial-mesenchymal transition in the mouse model of pulmonary fibrosis. J Cell Mol Med 20(7):1234-1246

Wang P, Wu T, Zhou H et al (2016) Long noncoding RNA NEAT1 promotes laryngeal squamous cell cancer through regulating miR107/CDK6 pathway. J Exp Clin Cancer Res 35(1):22

Willis BC, Borok Z (2007) TGF-beta-induced EMT: mechanisms and implications for fibrotic lung disease. Am J Physiol 293(3):525-534

Xie T, Liang J, Geng Y et al (2017) MicroRNA-29c prevents pulmonary fibrosis by regulating epithelial cell renewal and apoptosis. Am J Respir Cell Mol Biol 57(6):721-732

Yu F, Jiang Z, Chen B et al (2017) NEAT1 accelerates the progression of liver fibrosis via regulation of microRNA-122 and Kruppel-like factor 6. J Mol Med 95(11):1-12

Zhang X, Hong R, Chen W et al (2019) The role of long noncoding RNA in major human disease. Bioorg Chem 92:103214

Zheng X, Zhang Y, Liu Y et al (2018) HIF-2 $\alpha$ activated lncRNA NEAT1 promotes hepatocellular carcinoma cell invasion and metastasis by affecting the epithelial-mesenchymal transition. J Cell Biochem 119:3247-3256

Publisher's Note Springer Nature remains neutral with regard to jurisdictional claims in published maps and institutional affiliations. 